Article

\title{
Application of Miniaturized Sensors to Unmanned Aerial Systems, A New Pathway for the Survey of Polluted Areas: Preliminary Results
}

\author{
Francesca Fumian 1,2,*, Daniele Di Giovanni ${ }^{1,3}$, Luca Martellucci ${ }^{1}\left(\mathbb{D}\right.$, Riccardo Rossi ${ }^{1}(\mathbb{D})$ and \\ Pasqualino Gaudio ${ }^{1}$ (D) \\ 1 Department of Industrial Engineering, University of Rome Tor Vergata, 00133 Roma, Italy; \\ daniele.di.giovanni@uniroma2.it (D.D.G.); lc.martellucci@gmail.com (L.M.); \\ riccardo.rossi.en@gmail.com (R.R.); gaudio@ing.uniroma2.it (P.G.) \\ 2 Joint NBC Defence School of Rieti, 02100 Rieti, Italy \\ 3 Unicamillus-Saint Camillus International University of Health Sciences, 00131 Roma, Italy \\ * Correspondence: francesca.fumian@esercito.difesa.it
}

Received: 16 March 2020; Accepted: 3 May 2020; Published: 6 May 2020

\begin{abstract}
With the aim to have risk mitigation for people and first responders, active remote sensing standoff detection is a fruitful technology, both in case of accidental (natural or incidental) or intentional dispersion in the environment of volatile chemical substances. Nowadays, several laser-based methodologies could be put in place to perform extensive areal monitoring. The present study regards the proposal for a new system architecture derived from the integration of a low-cost laser-based network of detectors for pollutants interfaced with a more sophisticated layout mounted on an unmanned aerial vehicle (UAV) able to identify the nature and the amount of a release. With this system set up, the drone will be activated by the alarm triggered by the laser-based network when anomalies are detected. The area will be explored by the drone with a more accurate set of sensors for identification to validate the detection of the network of Lidar systems and to sample the substance in the focus zone for subsequent analysis. In this work, methodologies and requirements for the standoff detection and the identification features chosen for this integrated system are described. The work aims at the definition of a new approach to the problem through the integration of different technologies and tools in the operative field experiments. Some preliminary results in support of the suitability of the integration hypothesis proposed are presented. This study gives rise to an integrated system to be furtherly tested in a real environment.
\end{abstract}

Keywords: lidar; standoff system; air pollution remote sensing; unmanned aerial vehicles (UAV); detection chemical agent; identification chemical agent; volatile organic compounds (VOC)

\section{Introduction}

The standoff detection and identification of chemical threats is a clever methodology to allow continuous monitoring from a safe distance of sensitive areas, such as landfill and critical infrastructures. This solution gives the possibility to have a quick pre-alarm in case of an industrial misoperation, or tentative destruction by burning of solid illegal wastes, or dispersion in the open air of gaseous ones, avoiding direct contact of first responders with an unknown release. For several decades, standoff detection systems that operate in mid-infrared (MIR), the spectral region in which it is possible to detect chemical threats, have been developed. As reported in studies [1,2], many applications have been conducted in the field of explosives and chemical agents detection. This technique allows us to reach higher sensitivity and wider spatial range compared with a technique that works in the other parts of the spectrum, but it is unable to identify the nature of substances dispersed. 
Moreover, previous studies demonstrated that the Lidar technologies were fruitful both to detect in real-time particulate matter emitted in an urban area by vehicles, showing high sensitivity and responsivity [3], then for early detection of forest fires reducing false alarms due to obstacles on laser path $[4,5]$. Other studies that apply tunable laser and more complex optic systems such as dial technology had shown the possibility to identify a wide range of chemical substances but are more complex and expensive [6]. The Lidar system nowadays can be realized at relatively low cost, but the weakness of this technique is that it can only identify the macro-class of the substance. Thus, it is unable to identify a substance or discriminate a single substance in a mixture.

In fact, the currently commercialized laser-based systems are able to detect the putative variation of the environmental chemical background, due to chemical agents (CAs) such as volatile organic chemicals (VOC) or toxic industrial chemicals (TIC) in a restricted spatial range, providing an on/off response type (usually known as trigger systems), but not to perform their identification.

A possible approach to improve the detection capability of MIR Light Detection and Ranging (Lidar) technique is the creation of a network composed by low-cost detection systems, Lidar-based already tested in urban [3] and industrial areas to detect pollutants and emissions sources that could be used as a first alarm. Regarding the MIR Lidar identification limit, a possible solution is to interface this pre-alarm system, the network of MIR Lidar, with a mobile platform equipped with a set of sensors. This feature can be sent in the focus area identified by the Lidar network when a punctual identification is needed, and sampling is required as a confirmation. This allows having a qualitative and quantitative characterization of the event, aimed at analyzing the pollutants released.

The flying platform will be equipped with specific sensors, able to investigate and discriminate the nature of the threat for several classes of environmental contaminants. Some studies have been conducted on the use of gas sensors mounted on a drone [7]. In the field of gas leakage localization with mobile robots, different approaches have been compared, and a gas sensing system has been developed and tested for the localization and mapping of the VOC [8]. Several other applications demonstrated that drones are suitable to be equipped with gas sensors for environmental monitoring and surveillance operations [9-14]. To the author's knowledge, it is the first time that the combination of the areal detection feature of a Lidar network and the punctual identification and sampling capabilities applied to a drone has been proposed. Therefore, this study is aimed to evaluate a laser-based detection system integrated with a drone sensor platform to offset the constraint of the single system and improve environmental monitoring. Furthermore, the drone will be fitted with a sampling system able to collect evidence from different environmental matrices.

Figure 1 presents the schematic representation of the overall system and of its operational logic.
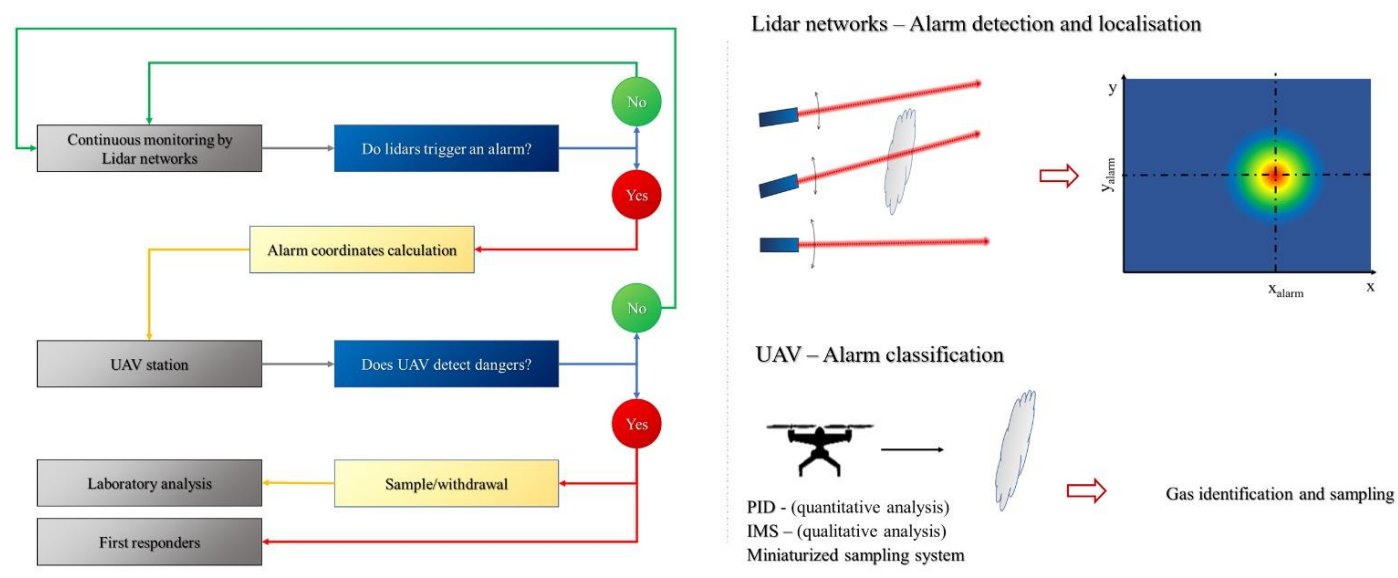

Figure 1. Schematic representation of the overall system and of its operational logic. 
The paper is structured as follows: Section 2 of Material and Methods gives an overview of the different technologies investigated, firstly the Lidar, as a standoff system for environmental monitoring and its possible use in a network as a method for environmental pollution detection. In the latter part of Section 2, UAVs state of the art is discussed to define a drone payload integrated with miniaturized sensors for chemical identification, such as Photo-Ionization Detectors (PID) and Ion-Mobility Spectrometry (IMS), and miniaturized sampling systems sensors as a method for pollutants identification. In Section 3, evidence of the preliminary results obtained is illustrated. Finally, in Section 4, regarding conclusions and future developments, the path for the integration of the whole system is defined.

\section{Materials and Methods}

In this section of the paper, techniques devoted to implementing specific functions in the overall integrated system proposed, such as detection, identification, monitoring, and sampling, were defined and described. After the description of each technique, the specific experimental setup chosen for its application in the system was investigated. Firstly, the early warning Lidar chosen as an areal and extensive detection method was presented. Secondly, an analysis of UAV and miniaturized environmental sensors identified as features for punctual monitoring was conducted. Finally, a suitable sampling solution was identified.

\subsection{Lidar}

As a basic concept, Lidar systems operate on similar working principles as the other 2 more familiar systems: Radar (radio detection and ranging) and sonar (sound navigation and ranging). Indeed, also the laser-based system is basically composed of a transmitter and a receiver, but, in the case of Lidar, a light pulse is transmitted into the atmosphere in the range of optical radiation (usually as ultraviolet, visible or near-infrared) instead of radio waves (Figure 2). The wavelengths choice for Lidar is specific for every application, and its range varied from about $250 \mathrm{~nm}$ to $11 \mu \mathrm{m}$ [15]. The light beam is scattered in all directions from molecules and particulates that beacon crossed through the atmosphere. The light portion that is backscattered toward the Lidar system is collected by the receiving optic that measures, as a distance function, the amount of backscattered light [16].

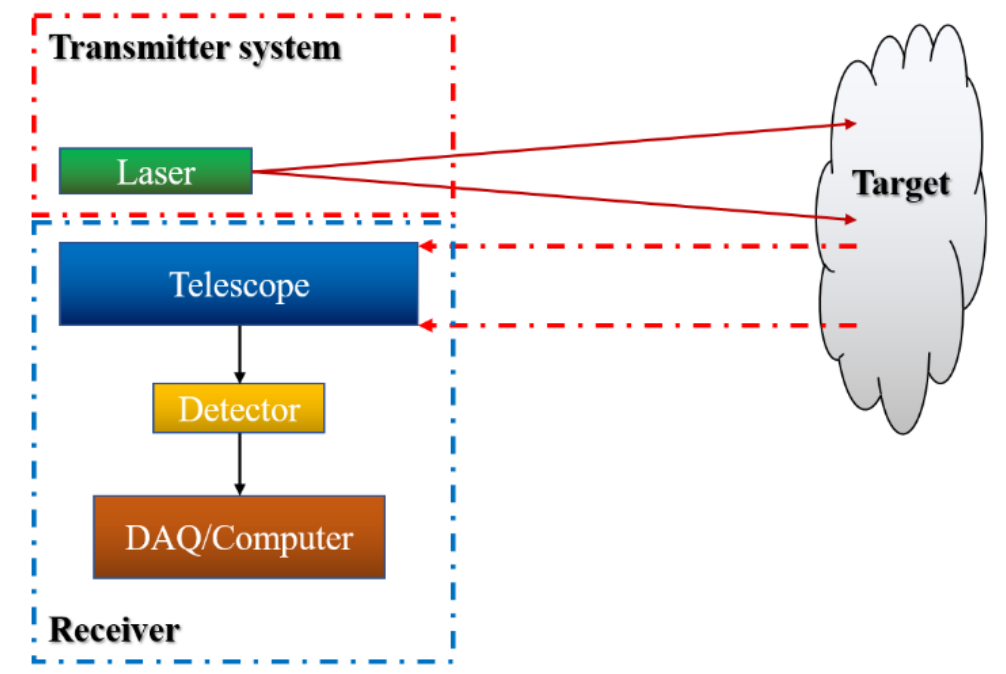

Figure 2. Conceptual scheme of a biaxial Lidar system.

Those systems could have additional components defined by the type and purpose of the Lidar. Moreover, it is possible to distinguish between bistatic and monostatic setup (Figure 3). 

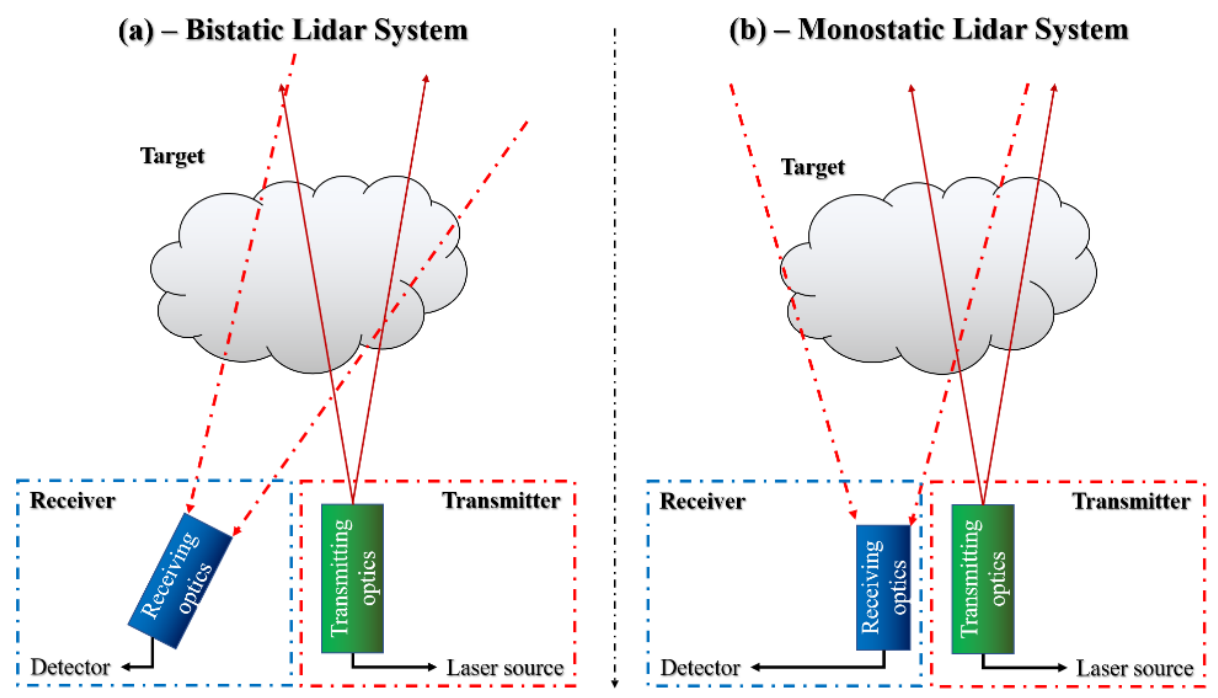

Figure 3. Bistatic (a) and monostatic (b) Lidar configurations.

In the monostatic configuration, the transmitter and the receiver are close in contact and aligned. This configuration is mostly used in modern Lidars in case of their use in the troposphere [17]. Conversely, when they are separated by a distance, the Lidar setup is called bistatic. In this last configuration, the common use is for long-distance or stratospheric applications.

It should be noted that the geometric arrangement of the emitter and receiver optics determines the degree of signal compression at distances close to the Lidar and, therefore, it can affect the final performances of a Lidar apparatus. In fact, at short distances, the laser beam cannot completely be imaged into the detector, and usually, only a part of the actual Lidar return signal is measured. Moreover, some practical adjustments can be adopted, such as interference filters placed in front of the detectors $[15,18]$, to suppress light outside the transmission band, for instance, to reset the background radiation.

\subsubsection{Lidar Standoff Systems for Environmental Monitoring}

In the last decades and in particular, after the first active remote sensing application [19] with pioneering studies of the upper region of the atmosphere, the lasers have played a fundamental role in environmental monitoring, and, in particular, they opened new fields with regard to remote sensing of the atmosphere. It is immediately appreciated that the Lidar, as with radar, could provide spatially resolved measurements in real-time. Lidar methodology has been plenty used to evaluate and detect profiles of pollutants emission. A system for the analysis of planetary boundary layer (PBL) dynamics [18] over the Naples city (Italy) was developed employing a self-aligning detection applied to the Lidar system operating at $351 \mathrm{~nm}$. The same application was carried out in order to evaluate PBL dynamics in Florence (Italy) [20]. This experiment had been the first example of a long-term monitoring study of urban aerosols with remote-sensing techniques. About urban pollutants, many studies have been conducted on UV Lidar measurements for the quantification of mass column density vehicle exhausts. Several studies reported similar concentration measurements, both in rural areas [21] than urban areas [22,23] and, moreover, the foundation of an aerosol Lidar network, EARLINET [24].

To sum up, laser-based techniques can be used to observe any density variation along the laser path as an irregularity on backscattered signals; variation that could be caused by the emission of pollutants in the atmosphere either by industrial releases. Because of these pieces of evidence, in recent time, the first use of plume automatic tracking has been proposed [25], for the evaluation of the particulate concentration and/or density variation as a function of the range/altitude of the ground with the capability to estimate the plume shape and its form. Those latest results showed the suitability of 
Lidar systems for plume tracking, definitively opening the use of the Lidar system for environmental monitoring to detect emission sources due to industrial releases and to the forest fires.

\subsubsection{Lidar Network as a Method for Environmental Pollution Detection}

The proposed system architecture included, as 1 of the 2 frames of the integrated system proposed, a network of sensors utilizing the Lidar technique, which is suitable for example on large agricultural or urban areas to monitor the presence of gas or particulate emissions, which could be caused by releases resulting from the normal ripening cycle of a given crop (e.g., ethylene for some fruit trees or color changes for "sick" crops) or uncontrolled releases of harmful substances or fire principles, giving, where necessary, the first alarm in almost "real-time".

For the network, creation has been chosen as a system developed at the University of Rome "Tor Vergata" [26] for the monitoring plume evolution from a forest fire and industrial flare. The compact Lidar system (COLI) consists of a compact, robust, and stable scanning mobile Lidar system based on monostatic configuration [17]. In Figure 4, the final configuration scheme is reported.

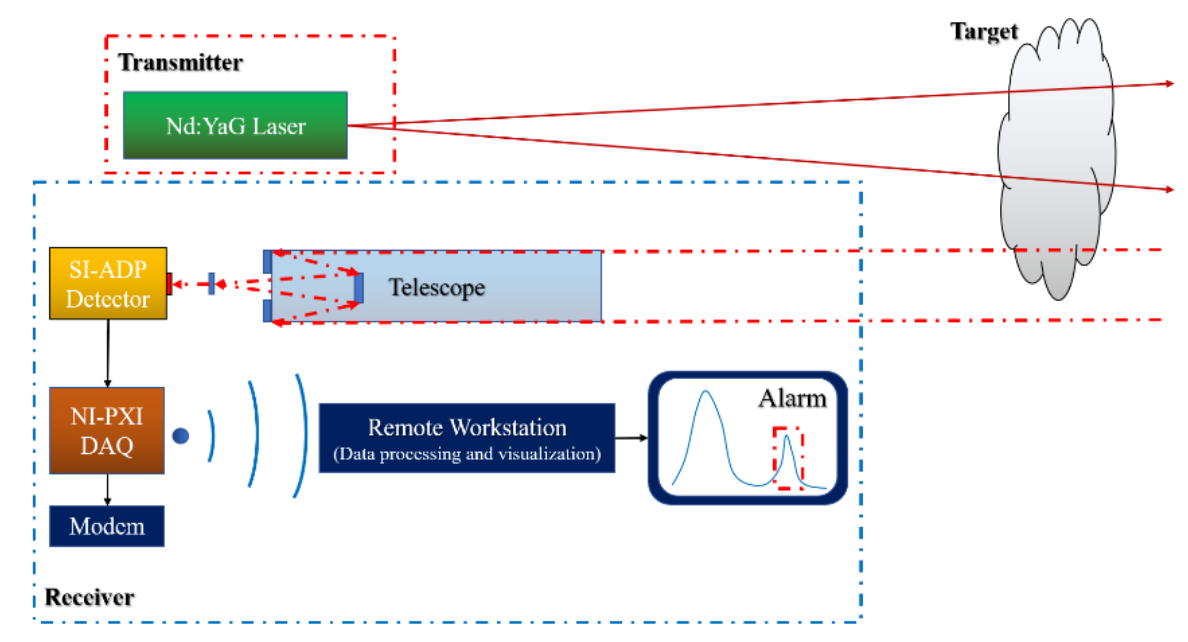

Figure 4. The technical scheme of the compact Lidar system (COLI) in its final configuration.

With this configuration, it is possible to scan the atmosphere in both vertical (elevation range from 0 to 90 degrees) and horizontal (azimuth range from 0 to 270 degrees) path, using computer-controlled motors incorporated into the laser-telescope rack. The spatial scan was automized and remotely controlled by self-designed software in LabVIEW, developed for mechanical handling of laser-telescope block and a MATLAB data acquisition and processing procedures. Experimental tests carried out to verify the effectiveness of the presented Lidar system were reported in the following section dedicated to the results.

\section{2. $U A V$}

In the last few years, advancements in the research and technological improvements, have been pushing the field of aerial robotics. A broad class of flying machines nowadays possess advanced position sensors and decision-making autonomy to accomplish complex tasks without the need for any direct human intervention. A wide range of flying platforms of different scale, mechanical configuration, and actuation principles exist $[27,28]$. This reflects the fact that for different scales, physical properties will lead to modified and novel design considerations $[29,30]$ with optimized endurance, agility, controllability, or perhaps simplicity [31].

Unmanned Aircraft Systems (which includes the UAV itself, the ground control system, camera, GPS, all the software, payload and skills needed to operate the system, and tools required for maintenance) (UAS), have drawn increasing attention recently, due to advancements in related 
research, technology, and applications. While having been deployed successfully in military scenarios for many years, civil use cases have lately been tackled by the robotics research community [27,32].

Given the capability and limitations on the payload, operational range, and power requirements of UAS, the choice of sensors, processors, and algorithms impose great technical and scientific challenges. Specifically, UAVs could represent one of the most suitable solutions in terms of system design, propulsion, perception, control, and navigation. However, different capabilities and properties arise, depending on their wings or flying mechanism: Between fixed and rotary wing UAV the appropriate configuration needs to be chosen based on the nature, target, and goals of the activity to accomplish [33-35].

A general classification of drones is done by their wing type [36]. "Fixed-wing" class has a very good battery life, can fly at a high altitude, and carry high weight. As for cons, it is expensive, can only move forward, and requires a good knowledge of aerodynamics for piloting. This category of drones does not grant the required proximity to operate specific sensors. The drones of the "single rotor" class, shaped like helicopters, are robust, and, compared to fixed wings, are able to hover and change direction easily than fixed wings. Moreover, these are less energy-consuming and have a good payload range. However, the piloting of this category of UAS is difficult, their wide propeller can be dangerous, and the turbulence could be a limitation for some kind of sensors. The last class is the most widespread and commercially available one: The "Multirotor drone". It is available in several configurations, sizes, and payload options. The main categorization is done by the number of motors [37], a higher number of rotor foster stability, the most common configuration is the quadri-copter [34], but also hexa-copter and octa-copter are frequently adopted. This class of drones can carry high payloads compared to their maximum take-off weight (MTOW). It is easy to maneuver, and, moreover, take-off and landing are possible almost anywhere. In addition, for a more stable flight, it can avail itself of an Inertia Measurement Unit (IMU), commonly equipped with gyroscope, compass, and accelerometer on the 3 axes. All these features allow the required proximity for the employment of chemical sensors and air sampling systems [38-40].

The advancements in the field of microprocessors, miniaturized sensing, as well as actuator efficiency and technologies downscaling, greatly improves the development of aerial robots. Moreover, the possibility to have autonomous guided UAS has to be considered, with the opportunity to proceed autonomously with a predetermined path and objectives based on the route descending from the alarm triggered by the Lidar. It is possible to conclude that in many cases, UAVs are more suitable with respect to a manned aerial vehicle, both in terms of cost and usability [38,39,41].

\subsubsection{UAV with Integrated Payload}

During recent years, we are withstanding constant examples of the development and diffusion of countless new uses of UAV in civil and military worlds, such as aerial photography, express shipments, and geographical mapping of inaccessible places. Furthermore, drones equipped with thermal sensors are nowadays almost currently used for search and rescue operations and collection of information during disaster management by first responders.

To fulfill the task of environmental monitoring, a flying platform must be equipped with several components to detect, identify, and possibly quantify the contamination occurred. Moreover, to confirm the assessment of the substances involved in the release, it is preferable also to foresee a sampling system for different kinds of substances within several matrixes.

One of the crucial aspects for monitoring of accidental or intentional release in the environment is the timing. In fact, once the release has been noticed, several preliminary activities to grant access to the hotspot must be completed. During this time, which may last for hours or days, the loss of evidence about the dynamics of the accident may occur, especially in case of strongly volatile substances. 


\subsubsection{UAV with Integrated Sensors as a Method for Pollutants Identification}

As we saw in the previous Lidar sections, the use of the Lidar system is not suitable to identify the substance released in the atmosphere. The idea behind this project is to combine the Lidar system for the areal monitoring and detection, with a secondary sub-system, an equipped drone, that acts as a sensor platform for the in-situ identification, monitoring, and sampling of the emission source and plume $[8,9]$.

The system requirements for the specific objective of this work have determined the platform choice. To lower the distance from the target and have an effective identification of the substance, the drone must be able to perform a high proximity flight to the release point identified from the Lidar. Furthermore, other crucial requirements are payload, energy consumption, flight autonomy to accomplish the mission, and stability to grant sensors operability. With these premises, the drone most suitable is a rotatory wing. In several previous studies, this configuration has been proven to give the right balance between autonomy, payload, and stability [8,10,41,42].

From the piloting point of view, to avoid the necessity of a professional aircraft pilot has been chosen to employ a mini-micro UAV category (maximum take-off weight MTOW: $25 \mathrm{~kg}$ ) as the most suitable solution.

\subsubsection{Miniaturized Sensors and Sampling Devices for Chemical Identification}

In the wide range of possibilities offered by the available working principles for chemical detection, 2 different technologies (PID and IMS) have been explored for this sub-system. By coupling those 2 working principles, it is possible to perform a qualitative and a quantitative assessment of the detected release jointly. Indeed, the first one put in place a working principle chosen for its reliability in assess concentration in the open air, its sensitivity, low weight, and low cost. The second one has been signposted for its capability to perform identification, with a given certainty at least for the class of chemical substances.

Other instruments, based on different working principles, were not investigated because they have been deemed too heavy or voluminous to be integrated on a mini category drone. Other ones were not considered because too expensive or not compatible with possible releases of combustible or explosive substances, for which the detector could become an ignition point.

For what regards the most suitable positioning of the sensors on the UAV, our research group was conducting studies by means of computational fluid-dynamic simulations. These studies evaluated the best compromise between the drone flyability and the influence that rotors turbulence had on the detection and identification capability. A specific study on the best sensors location in case of radioactive release detection had been already published [43], its pieces of evidence could be extended to the evaluations that have to be conducted about particulate matter pollution. A study about the evaluation of the best position of sensors for chemical detection dispersion is under conduction.

Photo-Ionization Detectors (PID)

The first working principle chosen for this application is photo-ionization detection (PID). Detectors using PID are effective in detecting and monitoring numerous hazardous substances and ware commonly low cost. Moreover, they provide a fast response [44]. Compared to several other methods of detecting dangerous gases available on the market, PIDs enclose a combination of response rates, ease of use and maintenance, compact size, and ability to detect low concentrations, including most volatile organic compounds (VOCs). PIDs used the working principle of CAs ionization. When the sample gas absorbs energy by a PID lamp, the gas gets excited, and its molecular content is altered. The compound loses an electron $\left(\mathrm{e}^{-}\right)$and becomes a positive ion [45]. Once this process occurs, the substance is considered ionized (Figure 5). 


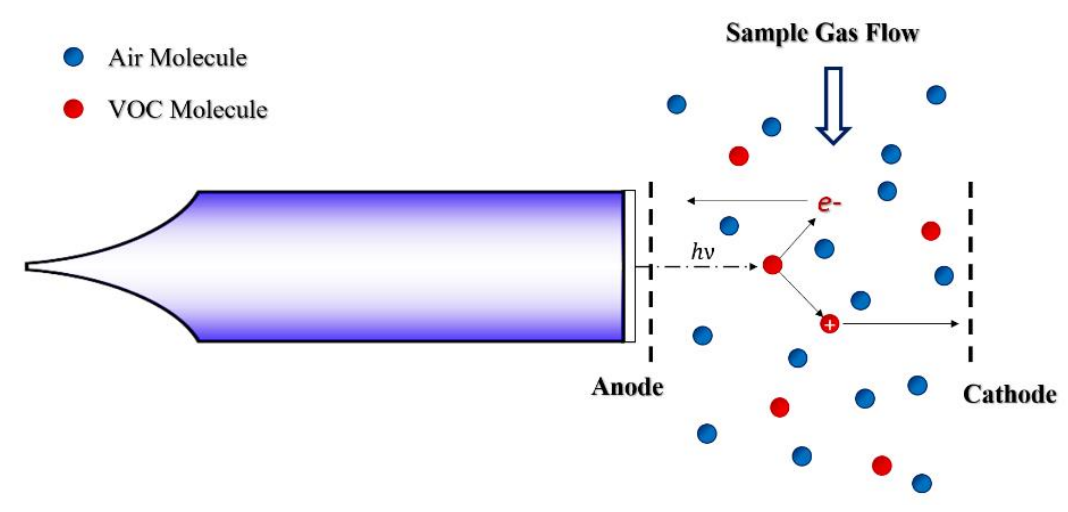

Figure 5. General schematic of a photo-ionization detection (PID) sensor.

Most substances can be ionized, some more easily than others. The aptitude of a substance to be ionized is measured as ionization potential (IP) with an energy scale in electron-volt (Ev). This scale generally ranges from a value of 7 to a value of about 16 , the higher the relevant value of IP, the more difficult it will be to ionize the substance.

To ionize the compound object of the monitoring, PIDs use an ultraviolet (UV) lamp. The lamp, which is often the size of a common flashlight bulb, emits at a specific voltage, for instance, $10.6 \mathrm{Ev}$ lamp emits enough UV energy to ionize any compound with an IP value less than $10.6 \mathrm{Ev}$. By measuring the current produced from the ionized compounds is possible to obtain its concentration as parts-per-million (ppm).

PID detectors can measure most of the organic compounds and some inorganic compounds, such as ammonia and sulfuric acid. The best way to calibrate the instrument is to expose it at a specific concentration of a certain compound, typically isobutylene, selected because it is located at a midpoint of ionization value for most of VOCs, moreover, it is not flammable or toxic at the concentration used for calibration. Once the instrument is calibrated, to conduct the measurement and obtain the concentration for a certain substance is necessary to convert the obtained result with a response factor (RF), that is compound specific. The RF is the ratio between the sensitivity of PID to the calibration gas compared to one of the gases that the user wants to measure. By the application of RF, it is possible to determine the concentration of a large number of compounds with a single gas calibration. Nowadays, databases of RF for many different chemical substances are freely available [46,47].

PID detectors are widely used in many different scenarios, such as leakage from industrial equipment, perimetral monitoring of industrial building, or storages whether the nature of spill is known, delimitation of contaminated areas after an emission, technical investigation after a fire. Therefore, due to their sensitivity and fast response, those instruments are extremely useful both for industrial applications for first responder's usage [48].

Therefore, PID detectors are able to measure the concentration of a known gas dispersed in the atmosphere, but they do not have identification capability. Indeed, they can be used to give a significative quantitative notice, but have to be coupled with an instrument with a working principle for the substance identification (qualitative evaluation).

Few applications have been previously conducted with the integration of those sensors with UAS, most of them devoted to combustion products [11,49] or particulate matter [12] investigation. For what regards industrial pollutants, evidence has been found of studies using electrochemical sensors [13], less sensitive than PID to VOC.

Regarding the future integration with a UAV suitable for this application, it has also been explored the need in terms of hardware to interface and collect sensor data. Among the PID sensors commercially available, Alphasense designs and manufactures sensors, such as optical monitors for aerosol and particulate matter (PM 10/PM 2.5), inorganic gases and VOCs with a limit of detection (LOD) of ppb, with a complete low-cost and lightweight microcontroller solution to program the sensor, process the acquired data and communicate it to the control station [50]. 
This solution presents a low power demand, both from the sensor than for the microcontroller, reducing further the overall payload of the drone. Furthermore, it allows the use in parallel an array of different detectors and features at the same time, controlled by the same hardware.

Among the several families of microcontrollers available on the market, one of the most popular is the Arduino series. It is mainly based on the Atmel AVR processor and provides many inputs and outputs in only one self-piece of hardware. Therefore, it offers flexible solutions for different needs, with a high-level language, cross-platform toolchain, and the capability of interface embedded devices. Hence, this family of microcontrollers will be the desirable choice to prototype the hardware and software interface with the sensors chosen for this application.

Ion-Mobility Spectrometry (IMS)

The second working principle selected for this application is Ion-Mobility Spectrometry (IMS). This is a well-established technology developed during the last century and widely applied in many types of detection instruments used as portable analyzers.

IMS detectors are selective equipment due to the different effects of the ionization process on different substances. Thus, they make it possible to discriminate different components in an air sample. The IMS working principle foresees 2 main stages. The first one is the sample reception and its ionization in the ionic reactor, with the creation of ions containing analyte molecules or their fragments. The second stage consists of the ion's separation, which occurs in the transducer part of the detector (Figure 6). The separation phase of ions distinguish IMS from other simpler ionization methods, such as flame ionization detectors (FID) and electron capture detectors (ECD) [51]. The output signal is generated by the transfer of the ions produced in the reactor towards the collector. This ion movement take place in a flowing stream of the carrier gas (advection) and in an electric field (drift).

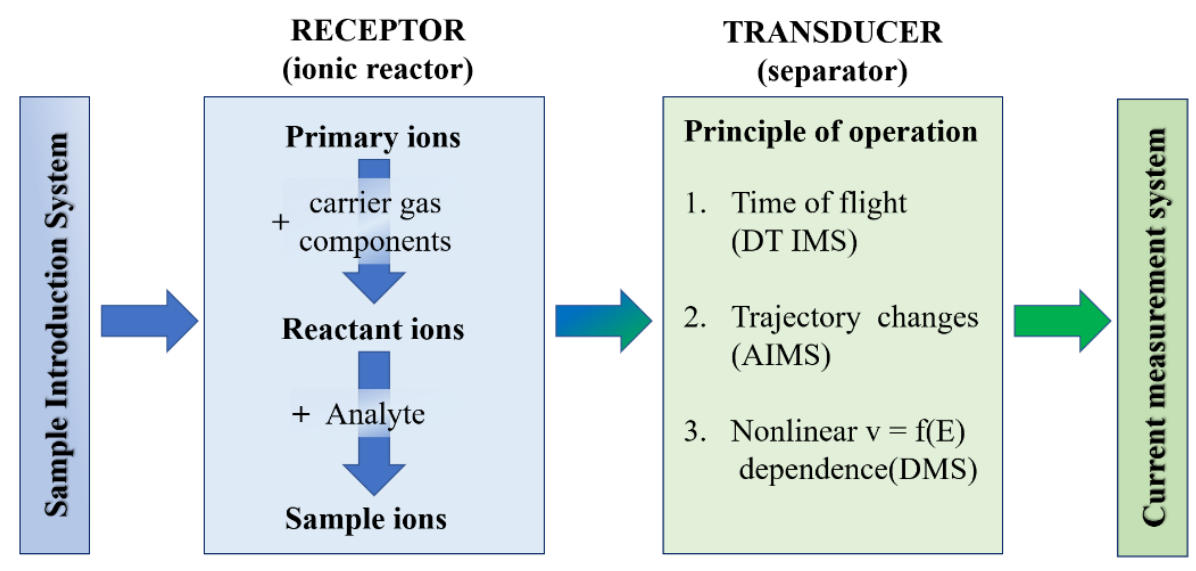

Figure 6. Ion mobility spectrometry (IMS)—operation principle and elements of measurement systems.

The process can generate positive or negative products on the gaseous sample volume withdrawn from the detector pump, employing different methods of ionization: Isotopic source emitting radiation, by a corona discharge (CD) [52] or UV [53].

In case of integration with a $\mathrm{UAV}$, radioactive sources such as ionizing agents are to be avoided, due to the regulation connected with aerial transport of dangerous goods [54].

Within the commercially available portable IMS detectors that employs corona discharge as an ionization method, the SMITH Detection instrument LCD3.3 could be a suitable solution. In a previous study, the same technology had been applied to evaluate an airborne methyl salicylate spread [14]. This instrument was AA battery-powered and performed continuous sampling and air analysis [55]. Selective and sensitive, the IMS, combined with the quantitative response given by the PID, can give a qualitative answer about the nature of the release. 
Miniaturized Sampling Systems for UAV Application

Technical specifications already spotted for the other sub-systems such as lightweight, plug-and-play function, low cost, and low energy consumption are still valid guidelines for the effective sampling system choice. In the field of environmental sampling, many different technological solutions are commercially available for the collection of significant and representative specimens. The main aim is to bring back a withdrawal to a laboratory from the location noticed by the Lidar network as the source of release and recognized from the other instruments integrated into UAS for the characterization and confirmation of the evidence. Assuming that the task will be absolved by the design of new equipment, it is proposed to realize a multi-sampling system working in parallel consisting of a device simultaneously CAs or unwanted TICs and particulate matter, preserving the characteristics of the sample until the time of analysis. The system will foresee specific sectors to selectively retain different substances, including sintered cartridge sampling systems [56], single-use or reusable after desorption, with the capability to intake samples from different environmental matrices, gaseous matrix, and aerosol samples.

Commercially available solutions with the indicated characteristics are air-sampler pumps. These devices are commonly used as personal equipment for environmental monitoring and providing low flows from 20 to $500 \mathrm{~mL} / \mathrm{min}$ for gas and vapor sampling, with the possibility of Bluetooth interface to program the sampling at a distance [57]. This equipment, with its restrained weight, commonly less than $250 \mathrm{~g}$, can employ different sampling media, such as impinger for powder trap or sintered metal cartridges able to immobilize VOC components and pollutants. The latest configuration gives the possibility to use a low flow tube holder to have many samples in parallel and have the possibility to replicate the analysis once the withdrawal is collected from the laboratory.

Regarding the integration of the sampling system to the UAS, the same interface used for PID detectors, an Arduino microcontroller, can be implemented to control in real-time duration, nature, and flowrate of the withdrawal.

\section{Preliminary Results}

This section presents some preliminary tests conducted to verify the functionality and effectiveness of some subparts of the proposed integrated system. The tests should be intended as the first phase of a wider testing campaign, which will lead, step by step, to assess the functionality of the environmental monitoring system as a whole (Lidar + multi-sensor UAV).

\subsection{Lidar Test Campaign}

Several field test campaigns have been conducted with the COLI system introduced in Section 2. These experimental works were intended both for the measurement of increasing in particulate matter due to vehicles [3], then for the detection and topographic identification of pollutant sources [58]. As a result of the first test campaign, a strong linear correlation of PM concentration levels has been evidenced between the laser-based detector and conventional monitoring station, with the possibility to reconstruct temporal maps of the monitored emissions.

In the second experimental campaign, a controlled fire was produced by the combustion of vegetables in a chimney located in an industrial area. The COLI system was sited at a distance of about $400 \mathrm{~m}$. The result of the temporal evolution of smoke plume emission by the described sources is shown in Figure 4 present in [59] where COLI signal's temporal maps vs. space and time acquired. Each scan has been reported in both the Cartesian (left side) and polar (right side) diagram. The red dotted boxes highlight the increase of backscattering level linked to the smoke fluctuations. The position change of red spots from the source of smoke even hundreds of meters is possibly due to wind blowing from North-West. This latter field tests demonstrated the capability of Lidar systems and, in particular of mini-Lidar COLI, to perform real-time assessment of tenuous smoke behavior in the free environment for a $1.5 \mathrm{~km}$ range. 


\subsection{Multisensor Drone Test Campaign}

Preliminary field tests have been conducted to assess the behavior of LCD3.3 detector on a rotatory wing UAS (Figure 7). Di-propylene glycol monomethyl ether [60] in the open field has been used to activate the GB (Whereas GB is the NATO acronym that identifies sarin nerve agent, an organophosphate characterized by high volatility) [61] agent channel of the sensor, the one devoted to organophosphates. These compounds are commonly used in agriculture as insecticides, but when used in concentrations exceeding the limit permitted by law, they can injure human health.

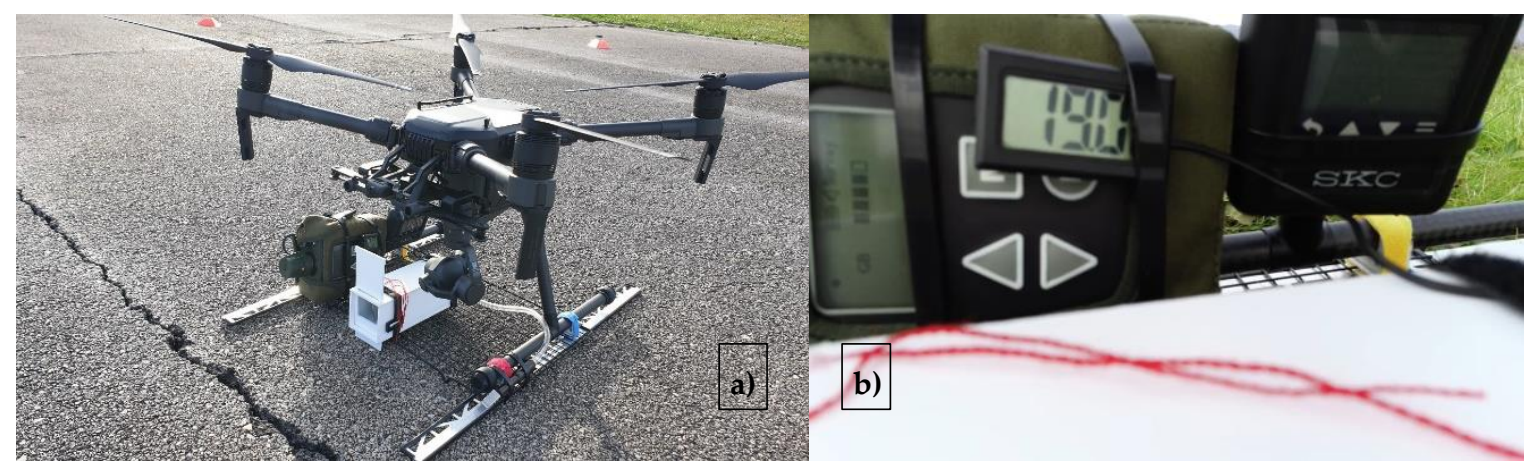

Figure 7. (a) The field test campaign arrangement; (b) the screen view of LCD3.3 detector on the alarm.

Connecting the instrument to a personal computer, it was possible to download the data-logs recorded every time an alarm had been activated. Figure 8 is the outcome of the data acquisition software Trimscan used to acquire results from the instrument. The spectrum plot and the spectrum data showed, for a single IMS channel, the peak position (drift time) on the spectrum giving note about the nature of detected CA. As it can be seen, the selected IMS instrument, that is commonly used for manned application by first responders and armed forces specialists, once integrated to a UAS, gave a good response in terms of detection. With a comparison of the altitude of the peak of the detected substance with the Reactant Ion Peak (RIP) was possible to give a rough quantification of the concentration of the agent in air.

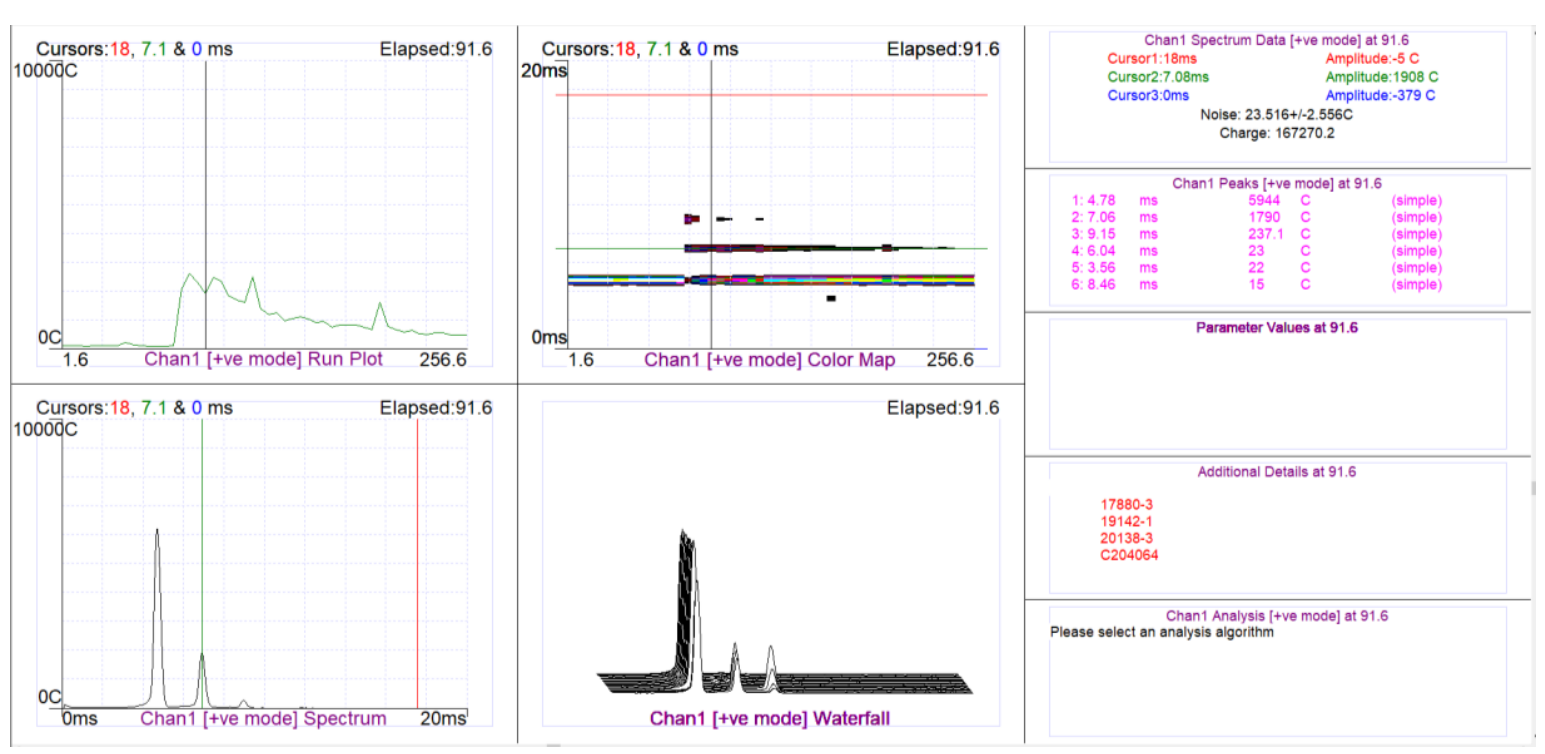

Figure 8. LCD3.3 trimscan reading of the alarm noticed in the field. 


\section{Conclusions and Future Developments}

At a time in history when we are witnessing malicious actions and accidents against the environment, the main objective of this research proposal is to develop a flexible, low-cost package, easily deployable in several scenarios. The overall layout has been conceived for a possible application to industrial contexts, critical infrastructures, landfills to be monitored, avoiding the illegal spillage of dangerous substances, or the attempt of destruction by fire of unauthorized wastes. The possibility of interfacing and intercommunicating different subsystems, each of which has a specific function, and broadens the range of possible missions to be carried out.

By going through the various subsystems illustrated, a low-cost network of Lidar sensors provides constant areal monitoring of large spaces and is responsible for raising an early alarm of a deviation from normal conditions. With this trigger, the UAV, equipped with proper sensors, is activated and sent in the location assessed by the laser-based coverage to be the origin of the release.

The two detection techniques identified to be suitable for this integration, PID and IMS, offer a good compromise between the several operational requirements pointed out. Indeed, their coupling is possible to perform, with good confidence, a quantitative (concentration with PID), and a qualitative (identification with IMS) measure of the detected release.

Moreover, the inclusion of a sampling system on the UAV makes it possible to collect significant samples for subsequent laboratory analysis and confirmation of the previous results obtained from field measurements.

In order to validate the goodness of the overall integrated configuration hypothesis experimentally, several test campaigns will be designed and carried out. The first group of measurements has already been conducted and was finalized to check the proper functioning of detection and identification features apart, giving the following preliminary achievements.

From the performed test with the COLI apparatus, it can be concluded that it represents a smart, compact, and low-cost standoff detection system. To control large critical areas, expected to be subject to aerial contamination and particulate matter spread, it is possible to use multiple COLI systems by integrating them into a network with a given mesh.

The preliminary tests conducted on the equipped flying platform give the evidence that the hypothesis of integration of the chosen instruments is suitable for this application and opens the horizon to new possible experimentations. Thus, the flying platform equipped with a light and low-cost payload that integrates sensors and sampling systems deployed on the emission site localized by the Lidar network allows solving the capability gap of Lidar in substances identification.

Moreover, the opportunity to collect samples of the environmental contaminants widens the possibility to confirm in-field measurements with laboratory analysis [62].

Future test campaigns will be conducted to assess the suitability of the integration of PID sensors and drones and to evaluate the interface of the Lidar network and the fully equipped drone.

Another crucial aspect to investigate is the position of the sensor on the UAS that has to be optimized in order to enhance detection and identification capabilities. A set of numerical fluid-dynamics simulations have already been conducted [43] and will execute a new set specifically dedicated to explore and understand the behavior of rotatory wings UAV turbulence with different environmental pollutants. This will allow performing in advance troubleshooting procedures to find out whether a configuration is viable.

Author Contributions: Conceptualization, F.F., D.D.G., L.M., P.G.; Formal analysis, D.D.G.; Funding acquisition, F.F.; Investigation, F.F.; Methodology, P.G.; Project administration, P.G.; Supervision, D.D.G.; Writing-original draft, F.F., L.M.; Writing-review \& editing, D.D.G., R.R. All authors have read and agreed to the published version of the manuscript.

Funding: This work has been partially funded by the Organization for Prohibition of Chemical Weapons (OPCW) with a grant received from the "Support for Research Programme" 2018. 
Acknowledgments: We would like to thank the Joint NBC Defence School of Rieti and its staff for their technical support in the development activities for the conduct of the experimental tests and Heli Protection Europe s.r.l. for their technical support in-flight activities and for providing the flying platform.

Conflicts of Interest: The authors declare no conflict of interest.

\section{References}

1. Goyal, A.K.; Myers, T.R. Active Mid-Infrared Reflectometry and Hyper Spectral Imaging. Laser-Based Optical Detection of Explosives; CRC Press: Boca Raton, FL, USA, 2015; pp. 168-212.

2. Sasic, S.; Ozaki, Y. Raman, Infrared, and Near-Infrared Chemical Imaging; Wiley: New York, NY, USA, 2010.

3. Parracino, S.; Richetta, M.; Gelfusa, M.; Malizia, A.; Bellecci, C.; De Leo, L.; Perrimezzi, C.; Fin, A.; Forin, M.; Giappicucci, F.; et al. Real-time vehicle emissions monitoring using a compact LiDAR system and conventional instruments: First results of an experimental campaign in a suburban area in southern Italy. Opt. Eng. 2016, 55, 103107. [CrossRef]

4. Gaudio, P. UMEL: A new regression tool to identify measurement peaks in Lidar/Dial system for environmental physics applications. Rev. Sci. Instrum. 2014, 85, 063112.

5. Gaudio, P. Application of a $\mathrm{CO}_{2}$ dial system for infrared detection of forest fire and reduction of false alarm. Appl. Phys. B 2007, 87, 373-378.

6. Rossi, R.; Ciparisse, J.; Malizia, A.; Gelfusa, M.; Gaudio, P. Multiwavelengh differential absorption lidar to improve measurements accuracy: Test with ammonia over a traffic area. Appl. Phys. B 2018, 148, 124-148.

7. Rosser, K.; Pavey, K.; FitzGerald, N.; Fatiaki, A.; Neumann, D.; Carr, D.; Hanlon, B.; Chahl, J. Autonomous Chemical Vapour Detection by Micro UAV. Remote Sens. 2015, 7, 16865-16882. [CrossRef]

8. Gas-Drone: Portable Gas Sensing System on UAVs for Gas Leakage Localization. Available online: http://3dom.fbk.eu/sites/3dom.fbk.eu/files/pdf/Rossi_etal_IEEE-gas_sensors_uav.pdf (accessed on 16 March 2020).

9. Karpowicz, J. Detecting Radiological, Biological and Chemical Threats with Drones. Available online: https://www.commercialuavnews.com/public-safety/detecting-radiological-biological-chemicalthreats-drones (accessed on 16 March 2020).

10. Nikolic, J.; Burri, M.; Rehder, J.; Leutenegger, S.; Huerzeler, C.; Siegwart, R. A UAV system for inspection of industrial facilities. IEEE Aerosp. Conf. 2013, 5. [CrossRef]

11. Bolla, G.; Casagrande, M.; Dal Moro, R.; Destro, M. ARIA: Air Pollutants Monitoring Using UAVs. Metro. Aero. Space 2018, 6. [CrossRef]

12. Bezantakos, S.; Schmidt-Ott, F.; Biskos, B. Performance evaluation of the cost-effective and lightweight Alphasense optical particle counter for use onboard unmanned aerial vehicles. Aero. Sci. Tech. 2018, 52, 385-392. [CrossRef]

13. Gu, Q.; Michanowicz, D.; Jia, C. Developing a Modular Unmanned Aerial Vehicle (UAV) Platform for Air Pollution Profiling. Sensors 2018, 18, 4363. [CrossRef]

14. Marinelli, W.; Schmit, T.; Dupuis, J.; Mulhall, P.; Croteau, P.; Manegold, D.; Beshay, M.; Lav, M. Cooperative use of standoff and UAV sensors for CBRNE detection. Proc. SPIE 2015, 5. [CrossRef]

15. Weitkamp, C. Lidar Range-Resolved Optical Remote Sensing of the Atmosphere; Springer: Berlin, Germany, 2005.

16. Kovalev, V.; Eichenger, W. Elastic Lidar: Theory, Practice and Analysis Methods; Wiley: New York, NY, USA, 2004.

17. Martellini, M.; Malizia, A. Cyber and Chemical, Biological, Radiological, Nuclear, Explosives Challenges, Cham; Springer: Berlin, Germany, 2017.

18. Measures, R. Laser Remote Sensing_Fundamentals and Applications; Wiley: New York, NY, USA, 1985.

19. Fiocco, G.; Smullin, L. Detection of Scattering Layers in the Upper Atmosphere (60-140 km) by Optical Radar. Nature 1963, 199, 1275-1276. [CrossRef]

20. Del Guasta, M. Daily Cycles in Urban Aerosol Observed in Florence by Means of an Automatic 532-1064 nm LIDAR. Atmos. Environ. 2002, 36, 2853-2865. [CrossRef]

21. Likar, K.; Eichinger, V. Monitoring of the Particles Above the Unpaved Road by Lidar Technique. Glob. Nest J. 2011, 13, 309-316.

22. He, T.; Stanič, S.; Gao, F.; Bergant, K.; Veberič, D.; Song, X.; Dolžan, A. Tracking of Urban Aerosols Using Combined LIDAR-Based Remote Sensing and Ground-Based Measurements. Atmos. Meas. Tech. 2012, 5 , 891-900. [CrossRef] 
23. Mei, L.; Brydegaard, M. Atmospheric Aerosol Monitoring by an Elastic Scheimpflug Lidar System. Opt. Exp. 2015, 23, A1613-A1628. [CrossRef]

24. Bösenberg, J. EARLINET: A European Aerosol Research Lidar Network. In Proceedings of the 20th International Laser Radar Conference (IPSL), Vichy, France, 10-14 July 2000; Available online: https: //www.mpimet.mpg.de/fileadmin/publikationen/Reports/max_scirep_348.pdf (accessed on 5 May 2020).

25. Andreucci, F.; Arbolino, M.; Sozzi, R. Lidar Utilisation for Industrial Stack Plume Automatic Tracking. II Nuovo Cim. C 1991, 14, 453-462. [CrossRef]

26. Quantum Electronics and Plasma Physics Research Group. Available online: www.qepresearch.it (accessed on 16 March 2020).

27. Feron, E.; Johnson, E.N. Aerial Robotics; Springer: Berlin, Germany, 2008.

28. International Conference on Modelling and Simulation for Autonomous Systems. Available online: https: //link.springer.com/conference/mesas (accessed on 16 March 2020).

29. Nonami, K. Autonomous Flying Robots: Unmanned Aerial Vehicles and Micro Aerial Vehicles; Springer: Berlin, Germany, 2010.

30. Beard, R.; McLain, T. Small Unmanned Aircraft: Theory and Practice; Princeton Univ. Press: Princeton, NJ, USA, 2012.

31. Siegwart, R.; Nourbakhsh, I.R.; Scaramuzza, D. Introduction to Autonomous Mobile Robots; MIT Press: Cambridge, MA, USA, 2011.

32. Siciliano, B.; Sciavicco, L.; Villani, L.; Oriolo, G. Robotics: Modelling, Planning and Control; Springer: Berlin, Germany, 2009; pp. 469-521.

33. Gurdan, D.; Stumpf, J.; Achtelik, M.; Doth, K.-M.; Hirzinger, G.; Rus, D. Energy-efficient autonomous four-rotor flying robot controlled at $1 \mathrm{khz}$. IEEE Int. Conf. Robot. Auton. Syst. 2007, 4. [CrossRef]

34. Hoffmann, G.; Huang, H.; Waslander, S.L.; Tomlin, C. Quadrotor helicopter flight dynamics and control: Theory and experiment. AIAA Guid. Navig. Control Conf. 2007, 6. [CrossRef]

35. Tomic, T.; Schmid, K.; Lutz, P.; Domel, A.; Kassecker, M.; Mair, E.; Grixa, I.; Ruess, F.; Suppa, M.; Burschka, D. Toward a fully autonomous UAV: Research platform for indoor and outdoor urban search and rescue. IEEE Robot. Autom. Mag. 2012, 3, 46-56. [CrossRef]

36. Fahlstrom, P.; Gleason, T. Introduction to UAV Systems; John Wiley \& Sons: New York, NY, USA, 2012.

37. Naldi, R.; Forte, F.; Marconi, L. A Class of Modular Aerial Robots. IEEE Conf. Decis. Control Eur. Control Conf. 2011, 12. [CrossRef]

38. Mettler, B. Identification, Modeling and Characteristics of Miniature Rotorcraft; Springer: Berlin, Germany, 2002.

39. Achtelik, M.C.; Doth, K.M.; Gurdan, D.; Stumpf, J. Design of a multi rotor MAV with regard to efficiency, dynamics and redundancy. AIAA Guid. Navig. Control Conf. 2012, 9. [CrossRef]

40. A Temporal Logic-Based Planning and Execution Monitoring Framework for Unmanned Aircraft Systems. Available online: https://www.aaai.org/Papers/ICAPS/2008/ICAPS08-025.pdf (accessed on 16 March 2020).

41. Bachrach, A.; Prentice, S.; He, R.; Henry, P.; Huang, A.; Krainin, M.; Maturana, D.; Fox, D.; Roy, N. Estimation, planning, and mapping for autonomous flight using an RGB-D camera in GPS-denied environments. Int. J. Robot. Res. 2012, 31, 1320-1343. [CrossRef]

42. Marconi, L.; Naldi, R.; Gentili, L. Modelling and control of a flying robot interacting with the environment. Automatica 2011, 2047, 2571-2583. [CrossRef]

43. Marturano, F.; Ciparisse, J.F.; Chierici, A.; D’Errico, F.; Di Giovanni, D.; Fumian, F.; Rossi, R.; Martellucci, L.; Gaudio, P.; Malilzia, A. Enhancing Radiation Detection by Drones through Numerical Fluid Dynamics Simulations. Sensors 2020, 20, 1770. [CrossRef] [PubMed]

44. MSA Safety. Available online: www.MSAsafety.com (accessed on 16 March 2020).

45. The PID Handbook-Theory and Applications of Direct-Reading Photoionization Detectors (PIDs). Available online: https://www.amazon.com/Handbook-Theory-Applications-Direct-ReadingPhotoionization-Detectors/dp/0976816210 (accessed on 16 March 2020).

46. Alphasense. AAN 305-06 VOC Correction Factor. Available online: http://www.alphasense.com/index.php/ safety/application-notes/ (accessed on 16 March 2020).

47. RAESYSTEMS. Chemical-Warfare-Agent-Measurements-By-PID. Available online: https://www.raesystems. com/sites/default/files/content/resources/Technical-Note-159_Chemical-Warfare-Agent-Measurements-ByPID_03-06.pdf (accessed on 16 March 2020). 
48. RAESYSTEMS. SensorRAE Electrochemical Conditioning Station. RAE SYSTEMS. Available online: https://www.raesystems.com/sites/default/files/content/resources/Manual_SensorRAE_UserGuide_0324006-000_RevB.pdf (accessed on 16 March 2020).

49. ELCOD Project Contribution. 2019. Available online: http://www.elcod.eu/wp/wp-content/uploads/2019/07/ 2019_elcod_5_pmeier.pdf (accessed on 16 March 2020).

50. Alphasense. Available online: http://www.alphasense.com/index.php/air/products/ (accessed on 16 March 2020).

51. Puton, J.; Namieśnik, J. Ion mobility spectrometry: Current status and application for chemical warfare agents detection. TrAC 2016, 85. [CrossRef]

52. Hill, C.A.; Thomas, C.L.P. A pulsed corona discharge switchable high resolution ion mobility spectrometer-mass spectrometer. Analyst 2003, 128, 55-60. [CrossRef]

53. Baumbach, J.I.; Sielemann, S.; Xie, Z.; Schmidt, H. Detection of the gasolinecomponents methyl tert-butyl ether, benzene, toluene, and $\mathrm{m}$-xylene using ion mobility spectrometers with a radioactive and UV ionization source. Anal. Chem. 2003, 75, 1483-1490. [CrossRef]

54. IAEA. IAEA Regulations for the Safe Transport of Radioactive Material. Available online: https://www.iaea.org/ newscenter/news/updated-iaea-publication-regulations-for-the-safe-transport-of-radioactive-material (accessed on 16 March 2020).

55. Smiths Detection Ltd. Technical Information LCD3.3. 2017.

56. Parker Balston, Gas and Liquid Sample Analyzer Filters-Sample Filters. Available online: http://www. balstonfilters.com/products/sample-analyzer-filters (accessed on 6 May 2020).

57. SKC INC. Available online: https://www.skcinc.com/catalog/index.php?cPath=100000000_101000000_ 101000550 (accessed on 16 March 2020).

58. Gaudio, P.; Gelfusa, M.; Richetta, M. Preliminary Results of a Lidar-Dial Integrated System for the Automatic Detection of Atmospheric Pollutants. Proc. SPIE 2012, 8534. [CrossRef]

59. Gaudio, P.; Malizia, A.; Gelfusa, M.; Murari, A.; Parracino, S.; Poggi, L.; Lungaroni, M.; Ciparisse, J.; Di Giovanni, D.; Cenciarelli, O.; et al. Lidar and Dial Application for Detection: A Proposal to Improve Safety and Security. J. Instrum. 2017, 12. [CrossRef]

60. Ouvry, Chemical Simulants for CBRNE. 2003-2020. Available online: https://www.ouvry.com/en/chemicalsimulants-for-bcrne/ (accessed on 16 March 2020).

61. Bartelt-Hunt, S.; Knappe, D.; Barlaz, M. A Review of Chemical Warfare Agent Simulants for the Study of Environmental Behavior. Available online: https:/www.tandfonline.com/doi/abs/10.1080/10643380701643650 (accessed on 16 March 2020).

62. Grasping from the Air: Hovering Capture and Load Stability. Available online: https://www.eng.yale.edu/ grablab/pubs/Pounds_ICRA2011.pdf (accessed on 16 March 2020). 